\title{
METHODS AND SOFTWARE ENGINEERING TOOLS FOR SIMULATION OF ROBOT DYNAMICS
}

\author{
J.AUZINSH, P.SLIEDE \\ Riga Technical University \\ 1, Kalku Str., LV-1658 \\ Riga, Latvia
}

\begin{abstract}
Methods and appropriate engineers -oriented tools DINA and LMS have been developed for the automated simulation of flexible robot dynamics, including those with closed kinematic loops and changing structure. The typical example of such mechanism is robot, performing assembly operations in industry or construction.
\end{abstract}

\section{Introduction}

The current trend is to leave to engineers the informal part of dynamic systems designing process, namely, the problem formulation and interpretation of the results.

The spatial mechanisms with closed loops, elastic bodies, one-sided constraints are examined. The structure of mechanisms can change (putting on or removing of additional geometric or kinematic constraints), if interaction with other objects takes place. Mechanisms can contain complex systems of drive and control, which include digital and analogue elements. The kinds of tasks to be solved include direct and inverse geometry, kinematic and dynamic analysis as well as oscillation form and frequency analysis. Together with well-known methods, such as constraint equations on the acceleration level with stabilization, the recursive Newton-Euler algorithm etc., original approaches, which allow to increase the simulation speed on both IBM PC's and parallel processing computers, are implemented.

\section{Used Methods}

\subsection{DYNAMIC MODEL IN THE IMPLICIT FORM}

If we assume that $q$ is a $n$-size vector-column of generalized coordinates of a mechanism, then the equations of motion are of the form:

$$
A(q) \ddot{q}+B(q, \dot{q})-Q(q, \dot{q}, p, t)=0
$$


where $A(q)-n \times n$ matrix of inertia; $B(q, \dot{q})$ - vector-column of generalized gyroscopic forces; $Q(q, \dot{q}, p, t)$ - vector-column of generalized applied forces; $p$ - $l$ - size vector of driving system variables (currents, voltages, pressures etc.).

The geometric constraints between bodies are of the form

$$
f_{i}(q)=0, \quad(i=1,2, \ldots, m) .
$$

Coordinates $g$ can be displacements in translation or rotational pairs of mechanism with tree structure, $f$ - constraint equations for closing kinematic loops. Taking into consideration of constraints together with the dynamic equations for an unconstrained system (Newton-Euler equations for single bodies or equations of an unclosed branched kinematic chain) yield a differential-algebraic equation system, the solution of which involves heavy problems of numeric stability (see [1]). Particular difficulties are encountered if the constraints are one-sided, e.g., during the time of bodies meeting and separating. In this case violation of constraints frequently occurs, which gives rise to tremendous accelerations and a process of in reality non-existing vibrations. Our practical experience suggests that the constraints should preferably be assigned to the derivatives with a stability according to Baumgarte [2]:

$$
\left\{\begin{array}{l}
A \ddot{q}+B-Q-C^{T} \lambda=0, \\
C \ddot{q}+\dot{C} \dot{q}+k_{1} \dot{f}+k_{2} f=0,
\end{array}\right.
$$

where $C=\frac{\partial f}{\partial q}-m \times n$ Jacobi matrix of additional constraint equations (2);

$\lambda$ - Lagrange multipliers - $m$ - size vector of additional constraint forces;

$k_{1}, k_{2}$ - coefficients for constraints stabilization [2].

The algorithms for developing an automatic dynamic model, which enable one to calculate the left sides of the constraints equations (2), also allow to calculate their first and second derivatives which are contained in equation (3), without using numerical differentiation [3].

A traditional way to determine the movement is to find the matrices and vectors $A, B, C, \dot{C}, Q, f, \dot{f}$ and solve a set of $n+m$ linear algebraic equations in regard to $\ddot{q}$ and $\lambda$ by formalized description of kinematic scheme, if $q, \dot{q}, p$ are known. Such a procedure must be performed repeatedly at each step of integration and this slows down the simulation process. The idea to consider the equations of motion in their natural implicit form is used in programs DINA and LMS :

$$
H(q, \dot{q}, \ddot{q}, p, t)=0
$$

where $H=\left[\begin{array}{c}H_{q} \\ H_{c}\end{array}\right]$ - left sides of the equations of motion and constraits.

In the case of opened branched kinematic chains it is quite easy to calculate the discrepancy of dynamic equations $H_{q}$ and the discrepancy of additional equations $H_{c}$ by the recursive principle of Newton-Euler equations without calculation of $A, B, C, \dot{C}$. It 
requires computing time proportional to $n$ [3]. Using, for example, the absolute stable implicit trapeze (Newmark) method for numerical integration:

$$
\left\{\begin{array}{l}
q^{j+1}=q^{i}+h \dot{q}^{i}+\frac{h^{2}}{4}\left(\ddot{q}^{i}+\ddot{q}^{j+1}\right) \\
\dot{q}^{i+1}=\dot{q}^{i}+\frac{h}{2}\left(\ddot{q}^{i}+\ddot{q}^{j+1}\right)
\end{array}\right.
$$

together with equations (4) gives us a set of non-linear equations in regard to $\ddot{q}^{i+1}$ and $\lambda^{i+1}$. (Index $i$ means the values of variables at the $i$-th moment of time $t_{i}=t_{0}+i h, h$ step of integration). A Newton type iteration method can be used to solve those equations. Doing only one iteration in each step with $k$ times less step proves more efficient than doing $k$ iterations. Thus we obtain the following formula of iteration :

$$
\left[\begin{array}{l}
\ddot{\boldsymbol{q}}^{i+1} \\
\lambda^{i+1}
\end{array}\right]=\left[\begin{array}{l}
\ddot{\boldsymbol{q}}^{i} \\
\lambda^{i}
\end{array}\right]-S \boldsymbol{H}\left(\boldsymbol{q}^{i}, \dot{\boldsymbol{q}}^{i}, \ddot{\boldsymbol{q}}^{i}, \boldsymbol{p}^{i+1}, t_{i+1}\right),
$$

where $S$ is $(n+m) \times(n+m)$ matrix

$$
S=\left[\frac{\partial H}{\partial \ddot{q}}+\boldsymbol{h} \frac{\partial H}{\partial \dot{q}}+\frac{h^{2}}{4} \frac{\partial H}{\partial \boldsymbol{q}} \mid \frac{\partial H}{\partial \lambda}\right]_{\mid t=t^{*}}^{-1} .
$$

It is also necessary to calculate matrices $A=\partial \boldsymbol{H}_{q} / \partial \ddot{\boldsymbol{q}}, \boldsymbol{C}=\partial \boldsymbol{H}_{c} / \partial \ddot{\boldsymbol{q}}$, but we need not do it in every step of the integration, because the configuration of the mechanism changes relatively slowly and, once calculated, matrix $S$ in fact guarantees convergence of (6) for at least further 100 steps of integration. Besides that we can take into account only the great components of matrices $\partial H_{q} / \partial q$ and $\partial H_{q} / \partial \dot{q}$ from the stiff springs and the dampers in joints:

Thus $S$ can be used in the form:

$$
\frac{\partial \boldsymbol{H}_{\boldsymbol{q}}}{\partial \boldsymbol{q}} \approx-\frac{\partial \boldsymbol{Q}}{\partial \boldsymbol{q}}, \frac{\partial \boldsymbol{H}_{\boldsymbol{q}}}{\partial \dot{\boldsymbol{q}}} \approx-\frac{\partial \boldsymbol{Q}}{\partial \dot{\boldsymbol{q}}} .
$$

$$
S=\left[\begin{array}{cc}
A+D_{1}+D_{2} & C^{T} \\
C & 0
\end{array}\right]_{t=t^{*}}^{-1},
$$

where $D_{1}$ and $D_{2}$ are diagonal matrices of the stiffness and the friction coefficients. Moreover, the calculation of $S$ has been reduced to inversion matrices $A+D_{1}+D_{2}$ and $C\left(A+D_{1}+D_{2}\right)^{-1} C^{T}$. Those are symmetric, positively definite matrices, hence in the iteration process they can be replaced by their diagonals. It creates a possibility to do the integration process simultaneously for all $q$, if $\dot{q}$ is a vector-column of generalized speeds of a single unconstrained bodies system :

$\dot{g}^{T}=\left[v_{1 x}, v_{1 y}, v_{1 z}, \omega_{1 x}, \omega_{1 y}, \omega_{1 z}, \ldots, v_{N x}, v_{N y}, v_{N z}, \omega_{N x}, \omega_{N y}, \omega_{N z}\right]$. 


\subsection{CONSIDERING THE FLEXIBILITY OF BODIES}

Two techniques were used for considering body flexibility. The first approach is based on the finite element (FE) method to describe, for example, the distributed elasticity and inertia of flexible robot link. To obtain a simulation procedure demanding a reasonable expenditure of computing resources, the following steps are proposed.

Decomposition of motion. The full motion of a mechanism is considered as consisting of "rigid" one with superimposed "flexible" vibrational motion $[4,5]$. Description of the "rigid" motion is obtained by recording displacements in joints $\boldsymbol{q}_{i}$, velocities and accelerations of bodies (links) $\omega_{i}, a_{j}, \varepsilon_{i}$ during simulation of rigid mechanism by program LMS. "Flexible" motion is described as additional displacements of FE model nodes in coordinate frames, connected with bodies in "rigid" motion. Considering $n_{i}$ linear and angular displacements of the $i$-th body as vector $u_{i}$ with components, small enough to neglect some terms in expressions of inertia forces, caused by "rigid" transfer motions, the "flexible" dynamics of a separate link is represented by linear equations:

$$
M_{i} \ddot{u}_{j}+C_{i} u_{i}=R_{i},
$$

where $M_{i}, C_{i}$ - constant matrixes of inertia and stiffness of the body's FE model, $\boldsymbol{R}_{i}$ - a column-matrix of external loads, including inertia forces.

Reduction of FE model. As the size $n_{i}$ of the system (11) usually is too large for practical use, the reduction of the model is necessary. Various reduction techniques have been suggested, see $[6,7]$, but fulfillment of the following demands for reduced models are still actual:

1) the reduced equations system must be of minimal size $m_{i}$, using as variables a set of chosen displacements from unreduced FE model;

2) displacements in statics solution from applied forces $R_{i}$, inertia features of link as solid body and first $\boldsymbol{m}_{i}$ eigenfrequencies and vibration modes (eigenvectors) must be preserved in reduced model.

To fulfill these demands the approach, proposed in [10], is modified for enforced oscillations [9]. The vector $u_{i}$ is devided in retained part $u_{i 1}$ and omitted elastic freedoms $u_{i 2}$. After transformation

$$
y_{i}=\left[\begin{array}{c}
y_{i 1} \\
y_{i 2}
\end{array}\right]=\left[\begin{array}{c}
u_{i 1} \\
L_{i} u_{i 1}+u_{i 2}
\end{array}\right]
$$

where $L_{i}=-\Phi_{i 12} \Phi_{i 11}^{-1}$ - matrix with the first $m_{i}$ modal vectors of (11) as columns, the system (11) may be presented in the form:

$$
\begin{aligned}
& \ddot{y}_{i 1}+X_{i 11} y_{i 1}+X_{i 12} y_{i 2}=P_{i 1} \\
& \ddot{y}_{i 2}+X_{i 22} y_{i 2}=P_{i 2}
\end{aligned}
$$

As shown in [10], eigenvalues of $X_{i 11}$ correspond to $m_{j}$ lower eigenfrequencies and those of $X_{i 22}$ - to $n_{i}-m_{i}$ higher ones of unreduced FE model. Usually frequency 
spectrum of $R_{j}$ is sufficiently lower as these $n_{i}-m_{j}$ frequencies of $X_{i 22}$. As result (13b) gives

$$
y_{i 2} \approx X_{i 22}^{-1} P_{i 2}
$$

After returning to the variables $u_{i}$, the reduced model is obtained, which satisfies all demands, formulated above:

$$
\bar{M}_{j} \ddot{u}_{i 1}+\bar{K}_{i} u_{i 1}=R_{i 1}+\Gamma_{i} R_{i 2} \text {, }
$$

where expressions for $\bar{K}_{i}$ and $\Gamma_{i}$ are the same obtained from the method of static condensation, but

$$
\bar{M}_{i}=M_{i 11}-M_{i 12} L_{i}+\Gamma_{i}\left(M_{i 21}-M_{i 22} L_{i}\right),
$$

Considerable advantage of proposed reduction procedure is the possibility to obtain $L_{i}$, as shown in [8], by iterative solution of a Riccati matrix equation:

where

$$
L_{i} G_{i 11}-G_{j 22} L_{i}-L_{i} G_{i 12} L_{i}+G_{i 21}=0
$$

$$
G_{j}=M_{i}^{-1} K_{i} \text {. }
$$

"Assembly" of complete mechanism model. A formalized algorithm and program MON is developed to perform "assembly" of reduced models of links to obtain a model, which describes displacements of a chain, consisting of rigid and flexible bodies, from nominal "rigid" configuration, given by $\boldsymbol{q}_{j}$. Three constraints are imposed by each hinge - coincidence of displacements of hinge elements central points. Relative angular displacements in hinge appears as variables in complete model, one of them, $\mu_{i}$ is additional turning around the main axis of the hinge. As result the model of the whole mechanism is obtained in form of a linear equations system with time - dependent matrixes, determined by nominal position vector $q$ :

$$
M \ddot{U}+K U=R+F(q+\mu, \dot{q}+\dot{\mu}, p, \dot{p}),
$$

where $F$ is vector of torques of the drives.

Simulation. A special modification of the program LMS (named VMS) was developed for simulation of the model (19). The proposed FE approach and programs were successfully used for simulation of a large scale space manipulator after testing by comparison with results, obtained by in-cut fictitious joint method, described below as the second technique.

If the mechanism contains elastic beam-shaped bodies, then it is rather expedient to use the in-cut fictitious joint method by placing in them massless springs which simulate the body's elasticity. This method has been long known in literature, in the report [8] the term "superelement" is used to designate a combination of several such joints. For the replacements and turnings of a beam length $l$ caused by forces applied to its ends in statics to coincide with co-planar model of a beam, it is necessary to place into it two fictitious joints at equal distances from its ends, by dividing it into parts of lengths $a, b, a$ (see Fig. 1.), $a=(1-1 / \sqrt{12}) l$.The stiffness of the springs placed in 
the joints has to be $c=2 E I / l$. In this way $2 m$ in-cut joints can be accommodated in the beam. In the spatial case of the model, joints have to be placed also along other axis perpendicular to the beam, as well as the joints with axis along the beam for consideration of the torsion deformation of the beam (see Fig. 2.). Such a model of the beam in statics coincides precisely with its FEM model.
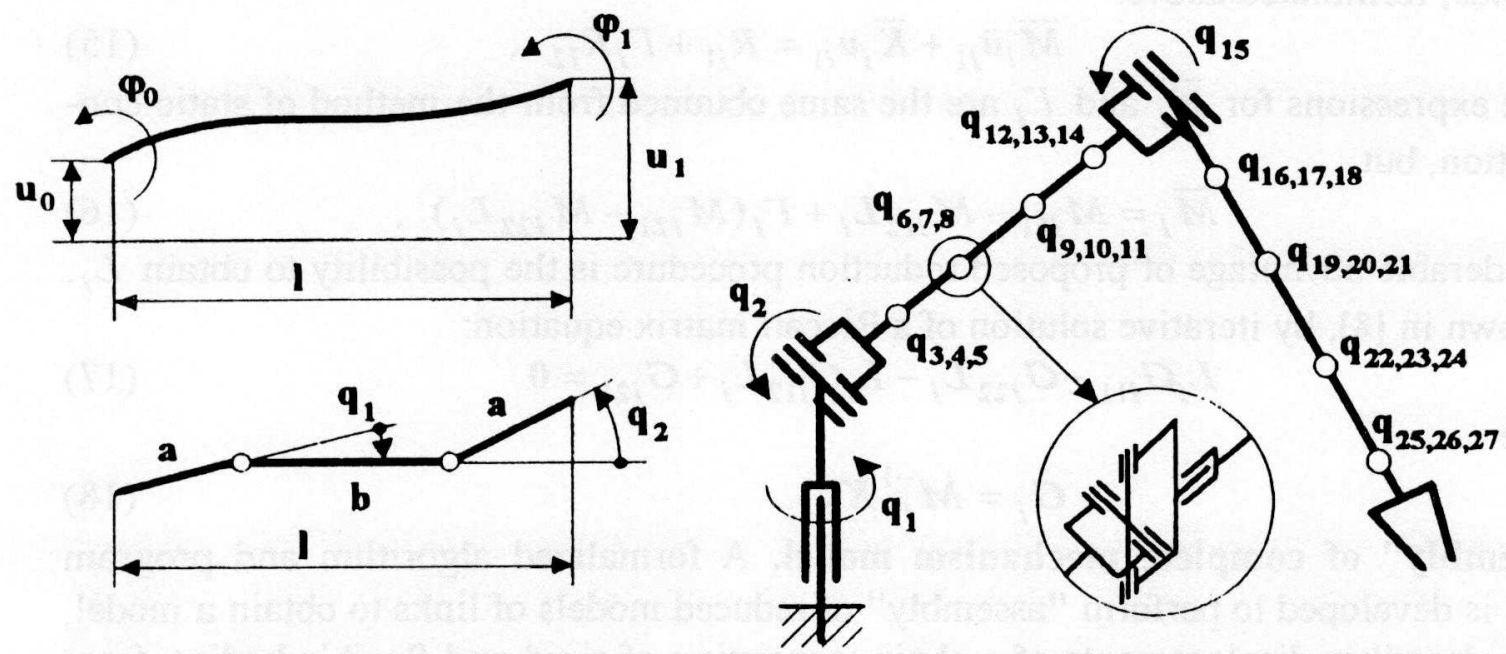

Fig. 1. Beam FE and rigid bodies model

Fig. 2. Flexible robot model

A test was conducted in which the eigenfrequencies for a console were compared under use of this method, of the "superelement" $a=l / 4$ used in [8] and FEM with the balanced inertia matrix. The method at issue, given an equal number of degrees of freedom (DOF), yields eigenfrequencies by one order more precise than FEM, with the exception just of the n-th frequency, which comes out somewhat more precise with FEM (see Table 1.). The beam pendulum with the mass concentrated at the end [8], gave analogous results.

Table 1.

\begin{tabular}{|c|r|r|r|r|r|r|r|r|r|}
\hline DOF & \multicolumn{3}{|c|}{$\omega_{1}$} & \multicolumn{3}{|c|}{$\omega_{2}$} & \multicolumn{3}{|c|}{$\omega_{3}$} \\
\hline & $\mathrm{A}$ & $\mathrm{B}$ & FEM & $\mathrm{A}$ & $\mathrm{B}$ & FEM & $\mathrm{A}$ & $\mathrm{B}$ & FEM \\
\hline 2 & 1.01 & 6.08 & .475 & 62.6 & 40.8 & 57.9 & - & - & - \\
\hline 4 & .018 & 1.45 & .048 & 2.77 & 5.21 & .848 & 16.4 & 7.49 & 80.4 \\
\hline 6 & .002 & .641 & .010 & .222 & 2.25 & .328 & 4.18 & 3.58 & 16.3 \\
\hline 8 & .0005 & .359 & .003 & .44 & 1.26 & .117 & .597 & 2.03 & 1.45 \\
\hline 10 & .0001 & .230 & .002 & .013 & .801 & .050 & .161 & 1.30 & 1.17 \\
\hline
\end{tabular}

Relative error $\%$ of beam eigenfrequencies. $A: a=221$; $B: a=25 l$; FEM: finite element method with balanced inertia matrix. 


\section{Software engineering tools and their applications}

DINA and LMS are broadly used for simulation of robots, cranes, aerials, artificial limbs etc. The unified pre- and postprocessors for all tools are used. Description of the objects, tasks and results can be stored in data base. All tools run on IBM and compatible PC under MS-DOS. DINA was successful in participating in the IAVSD organized tests [11] by simulating the dynamics of a five point wheel suspension.

The in-cut joint method just considered was used for simulation by means of a LMS program of a 3 DOF elastic robot (see Fig. 2.). Joints 1,2,15 are genuine joints, operative in which are PID-controlled drives, but for the others in-cut joints with springs and elements of linear friction. The total number of DOF is 27. Fig.3. present the graphs of some of the simulation results. Compared to the motion division method, the advantages are that repeated simulation is not required and such elastic systems can be simulated, in which the "hard" motion differs strongly from the elastic one. Because the deformations appear just as generalized coordinates but not as displacement differences as it is in the FEM models, smaller numeric rounding errors are obtained.

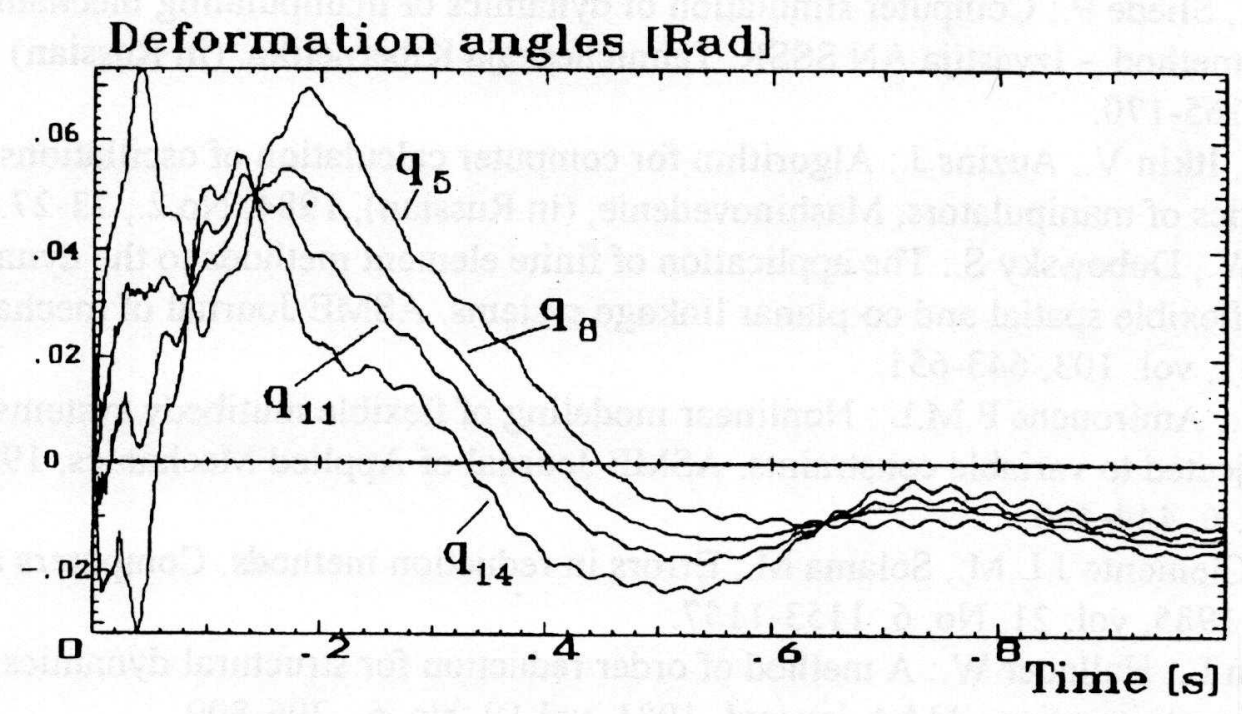

Fig. 3. Robot arm deformations

\section{Conclusions}

1. The problem of inverse dynamics (obtaining the motion by the active forces) is not to be reduced to calculation of the acceleration. Efficient algorithms can be obtained only if the formation of the dynamics model involves the method of numeric integration. 
2. If the algorithm is dominated mainly by the quantities which the constructors are interested in (the linear and angular coordinates of the bodies, the speeds and accelerations in the Cartesian coordinate system, the reactions and the driving forces in the joints, reactions on the contact points of the bodies etc.) and each of them is calculated from the quantities previously calculated by a minimum number of operations, the algorithm should be considered as conditionally optimal.

3. Interface with the corresponding optimization and identification codes provide a wide area of applications in calculations of controlled machine dynamics. Correspondingly well -grounded engineers decisions can be made with relatively low involved expenses.

\section{References}

1. Petzold L.R.: Differential-algebraic systems are not ODE's .-SIAM Journal on Scientific and Statistical Computing, vol. 3, No 3, 1982, 367-384.

2. Baumgarte, J.: Stabilization of constraints and integrals of motion. Computer Methods in Appl. and Eng. 1 (1972), 1-16.

3. Auzins J., Sliede P.: Computer simulation of dynamics of manipulating mechanisms by implicit method. - Izvestija AN SSSR. Tehnicheskaja Kibernetika. (in Russian) $1984, \mathrm{~N} 6,165-170$.

4. Sliede P., Itkin V., Auzins J.: Algorithm for computer calculation of oscillations characteristics of manipulators, Mashinovedenie, (in Russian), 1984, No 2., 23-27. 5. Sunada W., Dubowsky S.: The application of finite element methods to the dynamic analysis of flexible spatial and co-planar linkage systems. ASME Journal of mechanical design, 1981, vol. 103, 643-651.

6. Iden S.K., Amirouche F.M.L.: Nonlinear modeling of flexible mutibody systems dynamics subjected to variable constraints. ASME Journal of Applied Mechanics, 1989, vol. 56, No. 6. 444-450.

7. Utku S. Clemente J.L.M., Solama M.: Errors in reduction methods. Computers and Structures, 1985, vol. 21, No. 6, 1153-1157.

8. Anderson L., Hallauer W.: A method of order reduction for structural dynamics based on Riccati iteration, AIAA Journal, 1981, vol.19, No. 6., 796-800.

9. Sliede P.B.: Simulation of dynamics of robots with flexible actuating mechanisms. Information control problems in manufacturing technology. Robotics and flexible manufacturing systems. V IFAC/IFIP/IFORS Symposium. Preprints. Moscow, 1986, 426-431

10. Rauh, J., Schielen, W.: Various approaches for the modelling of flexible robot arms.: Refined dynamical theories of beams, plates and shells and their applications. Elishahoff, I.; Irretier, H.(eds.).Berlin/...:Springer, 1987, 420-429.

11. Kortüm W., Sharp R.S., De Pater A.D.: Application of multibody computer codes to vehicle system dynamics, Oberpfaffenhofen CCG (Germany), Webling, 1991, 300 p. 